\title{
Study of Micro-vision Calibration Technique Based on SIFT Feature Matching
}

\author{
Tao $\mathrm{Hu}^{1}$, Hui-lan $\mathrm{Wu}^{2}$, and Guodong $\mathrm{Liu}^{1}$ \\ ${ }^{1}$ Harbin Institute of Technology, Department of Automatic Measurement and Control, \\ Harbin, China \\ \{hutao, lgd\}@hit.edu.cn \\ ${ }^{2}$ Research Institute of Shanghai Academy of Spaceflight Technology, \\ No. 803, Shanghai, China \\ wh13003@163.com
}

\begin{abstract}
In the micro-vision system, precise calibration of the pixel equivalent is a prerequisite for accurate visual inspection. Traditional calibration process not only needs to take standard parts as the base, but also has strict requirements on their shape, manufacturing precision, placement and so on. Because of such shortcomings of traditional method, this paper gives an improved SIFT calibration algorithm for the pixel equivalent. Firstly, get the image's characteristic points before and after its' moving, using SIFT algorithm. Then filter the mismatched points through the second filtering algorithm so that the matching accuracy can be greatly improved. Compare moving distances of the characteristic point pixels to the reference distance of optical grating. Then accurate pixel equivalents can be obtained. Experiments show that the calibration algorithm is more accurate than traditional methods. So this algorithm can completely replace the standard-parts method in micro-vision system.
\end{abstract}

Keywords: Vision measurement, Calibration, Feature Matching.

\section{Introduction}

Measuring object size using CCD pixels as the scale is the core of vision measuring technology. So we need to acquire the actual size of CCD pixel. And because of the existence of optics system, the pixels' actual application size should be the one which is calculated through the optics system. During the application process of the vision system, an accurate enlargement of the optics system is hard to obtain because of many factors, just like the camera's relative position with CCD, and the camera's assembly quality differences. So, calibration of CCD pixel equivalent is the precondition and foundation of the measurement based on image [1-2].

Currently, standard-parts method is commonest among the calibration of CCD pixel equivalent. E.g.: figure 1(a) Standard mask. Test the standard part's geometrical shape, and then transmit its precise size to digital image. The precision of this method is subject to the standard part's accuracy of manufacture and the picture border's 
inspection quality. Moreover, it has strict requirements on the shape of standard part. In addition, the standard part's border must be parallel to the direction of CCD pixel. Or the distance between parallel lines will correspond to slant image's pixel number. And there will be angular misalignment in the demarcated pixel equivalent, as the picture 1 (b) shows. To optimize the calibration method, this paper presents an improved SIFT-based method to demarcate the pixel equivalent of Microscopic inspection system, according to its calibration characteristics. SIFT-Feature point matching method is based on image's local gradient features. It has better adaptability to image's lighting change, fuzziness and distortion. It doesn't need to detect the object's border. And it's not sensitive to object's shape.

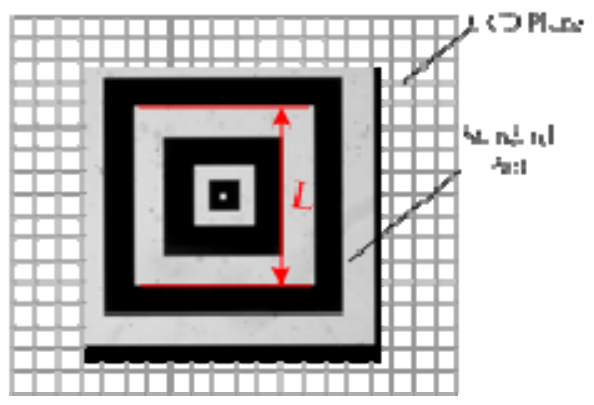

(a)

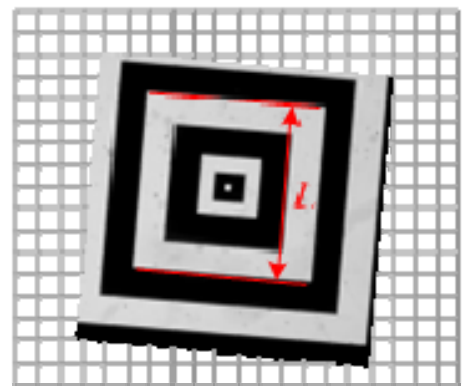

(b)

Fig. 1. Traditional method of calibration

\section{Calibration Technique}

Micro-vision system, which is based on SIFT- algorithm, can accomplish the calibration process with only the measured object. It doesn't have to draw support from standard part, which changes the traditional method that acquires the number of pixels of the reference distance. Detail calibration steps as follows: 1) Lay the object upon the one-dimensional translation stage; 2) Move the object. And collect the images of it separately before and after the moving; 3) Read the object's displacement $\mathrm{L}$ through the numerical reading device (optical grating or other displacement output unit); 4) Extract SIFT characteristic points of the collected images; 5) Match SIFT characteristic points; 6) calculate the number of pixel's corresponding distance the characteristic points moved; 7) $\mathrm{L} / \mathrm{N}$ is the pixel equivalent of the vision system.

What is key in the calibration method base on SIFT algorithm is the extraction and matching of SIFT characteristic points.

\subsection{Method of Abstracting SIFT Characteristic Points}

The SIFT algorithm extracts extreme points of Difference of Gaussian scale-space(DoG) as characteristic points, which mainly contains the following 4 steps [3-8]:

1) Build a DOG and extracts its extreme points;

$$
\begin{aligned}
D(x, y, \sigma)= & (G(x, y, k \sigma)-G(x, y, \sigma)) * I(x, y) \\
& =L(x, y, k \sigma)-L(x, y, \sigma)
\end{aligned}
$$


$G(x, y, \sigma)=\frac{1}{2 \pi \sigma^{2}} e^{-\left(x^{2}+y^{2}\right) / 2 \sigma^{2}}$ is a scale variable Gaussian function. $L(x, y, \sigma)$ is an

Gaussian image. $\mathrm{k}$ is a scale scaling factor. $\sigma$ is a Gaussian scale. The extreme point is the one that has the biggest change in grayscale value compared with its surrounding 26 pixels.

2) Precisely position extreme value points through fitting 3-d quadratic function.

3) Direction distribution of characteristic points

Calculate the images' gradient of characteristic points in the DoG. Determine the characteristic points' main direction by the way of accumulating their gradient. The amplitude and direction angle of gradient are calculated as follows:

$$
\left\{\begin{array}{c}
m(x, y)=\left[\begin{array}{l}
(L(x+1, y)-L(x-1, y))^{2} \\
+(L(x, y+1)-L(x, y-1))^{2}
\end{array}\right]^{1 / 2} \\
\theta(x, y)=\tan ^{-1} \frac{L(x, y+1)-L(x, y-1)}{L(x+1, y)-L(x-1, y)}
\end{array}\right.
$$

4) Build descriptor of the characteristic point

Turn the coordinate axis of Gaussian image $L(x, y, \sigma)$ to the main direction of characteristic points. The characteristic points being the center, divide its surrounding $16 \times 16$ pixel window into $4 \times 4$ windows. In each small window, accumulate the Gaussian image's gradient projection in 8 directions. Then a characteristic vector $\mathrm{F}$ of $4 \times 4 \times 8=128$ dimensions can be obtained.

\subsection{Characteristic Points Matching and Mismatch Filtering}

After detecting the SIFT characteristic points of the object's image, the points obtained after moving need to be matched with those before moving, thus confirm their one-to-one correspondence. The SIFT characteristic point matching methods that are in common used mainly include: NN(Nearest Neighbor), Exhaustive method, K$\mathrm{D}$ (K-Discriminator Tree), BBF(Best-Bin-First) and so on. Above methods are all based on traditional SIFT characteristic point descriptor, all getting too much error matching.

In the calibration process of micro-vision system, even a few mismatches can bring great deviation to the result. In order to overcome the influence of the mismatches, this paper does a second filtering to the mismatches, using the space coordinate information of characteristic points, which is based on NN(Nearest Neighbor) method.

Suppose $K\left(I_{\text {test }}\right)=\left\{k_{1}^{\text {test }}, k_{2}^{\text {test }}, \ldots k_{M}^{\text {test }}\right\}$ is the set of characteristic points of the image before object's moving. And $K\left(I_{\text {temp }}\right)=\left\{k_{1}^{\text {temp }}, k_{2}^{\text {temp }}, \ldots k_{M}^{\text {temp }}\right\}$ is the set of characteristic points of the image after object's moving.

Calculate the nearest and the second nearest distance $\left(M D_{i}\right.$ and $\left.S M D_{i}\right)$ between each couple of characteristic points' descriptor (before and after the image's moving). And then calculate the value: $S M D_{i} / M D_{i}$ as follows: . 


$$
\begin{gathered}
M D_{i}=\min _{j=1}^{M}\left(\operatorname{dis}\left(F\left(k_{i}^{\text {test }}\right), F\left(k_{j}^{\text {temp }}\right)\right)\right) \\
S M D_{i}=\sec { }_{j=1}^{M} \min \left(\operatorname{dis}\left(F\left(k_{i}^{\text {test }}\right), F\left(k_{j}^{\text {temp }}\right)\right)\right) \\
\operatorname{prop}_{i}=\frac{S M D_{i}}{M D_{i}}
\end{gathered}
$$

If $\operatorname{prop}_{i}>t h_{1}$, then point $k_{i}^{\text {test }}$ and point $k_{j}^{\text {temp }}$ are matching with each other. $t h_{l}$ is an appointed threshold value. With this step, usually $80 \%$ mismatches can be filtered. The larger th1 is, the less the number of matching couples is, and the better the stability is. But when $t h_{l}$ is too large, the number of matching point couples decreases so sharply that correct positioning is impossible, and not all mismatches can be eliminated. In practice, in most cases the value of th1 is around 1.5.

After initial characteristic point matching, point pairs can be obtained: $\left\{\left(s_{1}^{\text {test }}, s_{1}^{\text {temp }}\right),\left(s_{2}^{\text {test }}, s_{2}^{\text {temp }}\right), \ldots\left(s_{L}^{\text {test }}, s_{L}^{\text {temp }}\right)\right\}$, which still contains many mismatches. Because the relative position of characteristic points doesn't change after object's moving, a second matching can filter mismatches with the space coordinates information. Calculate the nearest distance $m d_{i}^{\text {test }}, m d_{i}^{\text {temp }}$ and the second nearest distance $s m d_{i}^{\text {test }}, s m d_{i}^{\text {temp }}$ separately within each characteristic point space.

$$
\begin{gathered}
m d_{i}^{\text {test }}=\min _{j=1, j \neq i}^{L}\left(\operatorname{dis}\left(s_{i}^{\text {test }}, s_{j}^{\text {test }}\right)\right) \\
s m d_{i}^{\text {test }}=\underset{j=1, j \neq i}{L} \sec \min \left(\operatorname{dis}\left(s_{i}^{\text {test }}, s_{j}^{\text {test }}\right)\right) \\
m d_{i}^{\text {temp }}=\min _{j=1, j \neq i}^{L}\left(\operatorname{dis}\left(s_{i}^{\text {temp }}, s_{j}^{\text {temp }}\right)\right) \\
s m d_{i}^{\text {temp }}=\sec \underset{j=1, j \neq i}{L} d \min \left(\operatorname{dis}\left(s_{i}^{\text {temp }}, s_{j}^{\text {temp }}\right)\right)
\end{gathered}
$$

If both $m d_{i}^{\text {test }} / m d_{i}^{\text {temp }}$ and $s m d_{i}^{\text {test }} / s m d_{i}^{\text {temp }}$ are smaller than the appointed threshold value $t h_{2}$, with $m d_{i}>10$ pixel ,then this couple of matching points be kept. If not, delete it. The value of $t h_{2}$ is about 0.9 in common case. As is in picture $2, k_{1}$ and $k_{3}, k_{2}$ and $k_{4}$ are all considered correct match before the second filtering. But

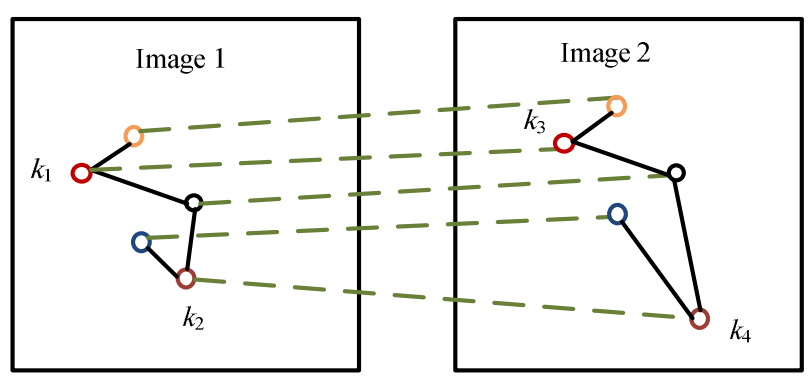

Fig. 2. Filter out the mismatches using space coordinates 
actually, only $k_{1}$ and $k_{3}$ are the correct couple. $k_{2}$ and $k_{4}$ are the mismatches. After the second filtering, only the couple of $k_{l}$ and $k_{3}$ will be retained.

Lots of experiments show that after the second time filtering, correct correspondence (before and after the object's moving) of object's SIFT characteristic points can be gained. And through affine transformation between images, the number of pixels corresponding to the SIFT characteristic points' moving distance can be calculated. With the reference displacement given, the pixel equivalent of micro-vision system can be demarcated.

\section{Experiments and Analysis}

In order to examine above ideology, this paper designed two experiments.

\subsection{Experiments of Standard Parts' Calibration and Comparison}

Firstly, lay a standard part (masking layer) upon the onedimensional electric transition stage which has a grating readout device. Resolution of the optical grating is $0.2 \mu \mathrm{m}$, using backlit lighting. CCD's type is

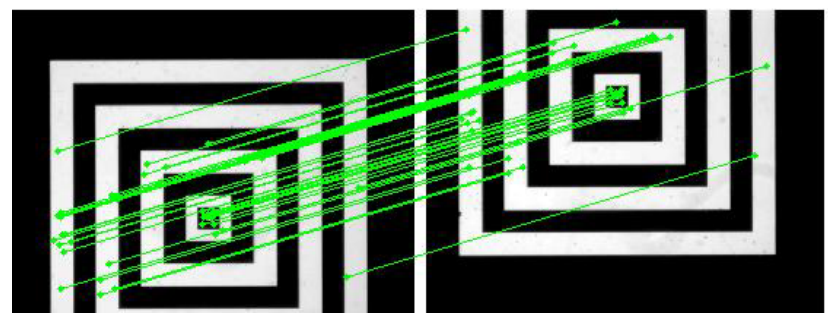

Fig. 3. SIFT matching point of the mask Japan's Watec $902 \mathrm{H}$, with a nominal pixel size value of $8.6 * 8.3 \mu \mathrm{m}$. To make a contrast, pixel equivalent calibration of the standard parts is done separately with the traditional method (including border detection, subpixel subdivision, parallel fitting) and this paper's method. Figure 3 shows the result of SIFT characteristic point matching. And table 1 shows the result of calculation.

Table 1. Calibration result of pixel using mask

\begin{tabular}{llllll}
\hline Number of experiments & 1 & 2 & 3 & 4 & 5 \\
\hline Grating indication $(\mu \mathrm{m})$ & 397.6 & 494.8 & 1044.4 & 1545.4 & 2192.2 \\
Distance of SIFT points (pixel) & 33.6978 & 41.9511 & 88.4361 & 130.7644 & 185.8332 \\
SIFT pixel equivalent $(\mu \mathrm{m} /$ pixel) & 11.7990 & 11.7947 & 11.8097 & 11.8182 & 11.7966 \\
Base space of mask $(\mu \mathrm{m})$ & 1000.0 & 2000.0 & 2000.0 & 3000.0 & 3000.0 \\
$\begin{array}{l}\text { Measured space of mask (pixel) } \\
\text { Pixel equivalent of traditional }\end{array}$ & 84.8 & 168.7 & 169.0 & 254.6 & 254.5 \\
method $(\mu \mathrm{m} /$ pixel) & 11.7925 & 11.8554 & 11.8343 & 11.7832 & 11.7878 \\
\hline
\end{tabular}


From table 1, the mean value of pixel equivalent is $11.8036 \mu \mathrm{m} /$ pixel with this paper's method. While with traditional method, the mean value is $11.8106 \mu \mathrm{m} / \mathrm{pixel}$. Being influenced by border detection's degree of precision, the result of traditional methods has less stability.

In order to check the accuracy of this paper's method further, move the standard part to a certain distance. The displacement can be read out by Renishaw ML10 twofrequency laser; Calculate the displacement's number of pixels with this paper's SIFT algorithm. And then calculate the actual displacement separately with above two calibration results. Table 2 shows the result.

As can be seen in table 2, the measuring result of this method is better than traditional method in application.

Table 2. Moving test error of mask image

\begin{tabular}{lccccc}
\hline Number of experiments & 1 & 2 & 3 & 4 & 5 \\
\hline $\begin{array}{l}\text { Displacement by Dual-frequency } \\
\text { laser }(\mu \mathrm{m})\end{array}$ & 494.6 & 504.2 & 507.4 & 498.7 & 495.1 \\
$\begin{array}{l}\text { Movement distance of } \\
\text { characteristic points (pixel) }\end{array}$ & 41.9270 & 42.7070 & 43.0163 & 42.2561 & 41.96 \\
$\begin{array}{l}\text { Error of pixel equivalent by mine }(\mu \mathrm{m}) \\
\begin{array}{l}\text { Error of pixel equivalent by } \\
\text { traditional method }(\mu \mathrm{m})\end{array}\end{array}$ & 0.2895 & -0.1037 & 0.3472 & -0.0741 & 0.1791 \\
\hline
\end{tabular}

\subsection{Experiment of Workpiece's Self-movement}

One obvious advantage of this calibration method is that the calibration algorithm is independent from object's shape. So the calibration can be done without standard parts, only making use of workpiece's self-moving. Keeping the same optical magnification as previous experiment, table 3 gives the

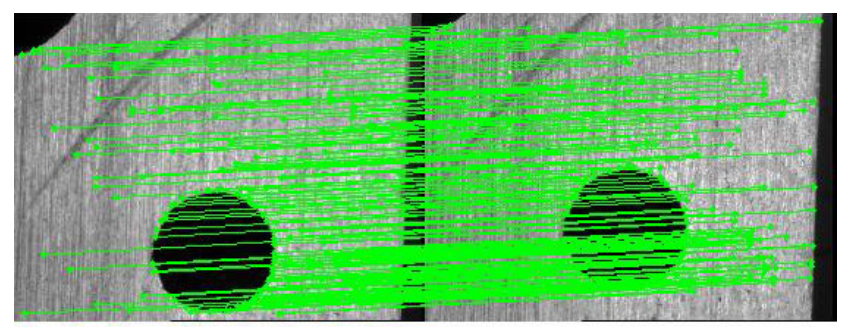

Fig. 4. SIFT matching point of the workpiece

Table 3. Calibration result 1 of work with a hole

\begin{tabular}{lcccccl}
\hline Number of experiments & 1 & 2 & 3 & 4 & 5 & mean error \\
\hline Pixel equivalent $(\mu \mathrm{m} /$ pixel $)$ & 11.7893 & 11.8225 & 11.8105 & 11.7934 & 11.8086 & \\
Error 1 of pixel equivalent $(\mu \mathrm{m})$ & -0.0213 & 0.0119 & -0.0001 & -0.0172 & -0.002 & -0.0057 \\
Error 2 of pixel equivalent $(\mu \mathrm{m})$ & -0.0143 & 0.0189 & 0.0069 & -0.0102 & 0.0050 & 0.0013 \\
\hline
\end{tabular}


results of vision system's calibration by workpiece with hole. Two images' matching result of SIFT characteristic points is given in figure 4. In table 3, pixel equivalent error 1 is the deviation of the two calibration results (the one that makes use of workpiece based on this paper's algorithm and the other by traditional method). Pixel equivalent error 2 is the deviation of the two results (the one that makes use of workpiece and the other that makes use of standard part, which all take this paper's algorithm).

Under different magnification, table 4 gives the calibration result of micro-vision system by workpiece with circular hole. In table 4, pixel equivalent error is the deviation of the two calibration results----the one that makes use of workpiece and the one with traditional method.

Table 4. Calibration result 2 of work with a hole

\begin{tabular}{lllllll}
\hline Number of experiments & 1 & 2 & 3 & 4 & 5 & mean error \\
\hline Pixel equivalent $(\mu \mathrm{m} /$ pixel $)$ & 8.7949 & 8.7997 & 8.7959 & 8.8017 & 8.7892 & \\
Error 1 of pixel equivalent $(\mu \mathrm{m})$ & 0.0052 & 0.0100 & 0.0062 & 0.0120 & -.0005 & 0.0066 \\
\hline
\end{tabular}

From table 3 and table 4, we can see that the calibration result by workpiece itself can maintain better repeatability with the method that uses mask. This further again proved this paper's method.

\section{Conclusion}

In micro-vision measuring system, calibration's precision of pixel equivalent applying traditional methods is subject to standard part. To overcome these shortcomings, this paper proposed a method based on improved SIFT local characteristic point matching to demarcate pixel equivalent. The experimental results show that the method presented in this paper has better stability than the traditional method. While reaching more stable calibration precision, the vision system's positioning accuracy is improved too. With this paper's calibration method, standard part is no more necessary in the process of microvision-detecting system's pixel equivalent calibration, overcoming the shortcoming that standard parts need to be positioned precisely.

\section{References}

1. Wu, J.-G., Bin, H.-Z.: Dimensional inspecting system of thin sheet parts based on machine vision. Optics and Precision Engineering 15, 124 (2007)

2. Wang, J., Pu, Z., Zhao, H., Lu, Z.: A new method for calibrating 2-D image measuring systems. Journal of Harbin Institute of Technology 32, 37 (2000)

3. Pan, X., Lyu, S.: Detecting image region duplication using sift features. In: Processing of IEEE International Conference on Acoustics, Speech and Signal, vol. 1706 (2010)

4. Bastanlar, Y., Temizel, A., Yardimci, Y.: Improved SIFT matching for image pairs with scale difference. Electronics Letters 46, 346 (2010) 
5. Li, J., Allinson, N.M.: A comprehensive review of current local features for computer vision. Neurocomputing 71, 1771 (2008)

6. Lowe, D.G.: Distinctive image features from scale-invariant keypoints. Int. Journal of Computer Vision 60, 91 (2004)

7. Mikolajczyk, K., Schmid, C.: A performance Evaluation of Local Descriptors. IEEE Transactions on Pattern Analysis and Machine Intelligence 27, 1615 (2005)

8. Wu, H.-L., Liu, G.-D., Liu, B.-G., Pu, Z.-B.: Study on the circle center fast accuratelocating technique based on the SIFT. Journal of Optoelectronics.Laser 19, 1512 (2008) 